\title{
Some Results of the Coordinated Observations of the Red Dwarf Flare Star EV Lac in 1986-1994
}

\author{
R.E. Gershberg \\ Crimean Astrophysical Observatory, Nauchny, Crimea 334413, Ukraine
}

In 1986-94 a series of coordinated monitorings for the flare star EV Lac has been performed. The observations were aimed to collect homogeneous data on the star both in its active and quiet states, to study its flares and starspots. These photometric patrols were accompanied by monitorings of EV Lac of different other kinds: polarimetric, spectral, radio, UV and high energy studies. In total, these monitorings covered a range from decametre radio wavelengths through IR, optical and UV windows up to very high energy $\gamma$-rays. The list of participants of these studies is very large. Up to date the results of 1986, 1987, 1990 and 1991 are published (Gershberg et al. 1991a,b, 1993, Alekseev et al. 1994), the data of 1989 are submitted (Berdyugin et al. 1995), the data of 1992 (Abdul-Aziz et al. 1995) will be submitted in the near future. In 1993, photometric observations were accompanied by monitoring observations of $E U V E$ and $I U E$, in 1994, VHE $\gamma$-ray observations were included. The publication of data collected in 1993 and 1994 is in preparation. In this communication I shall concentrate on several new findings related to the EV Lac flares only.

1. Colour-Colour diagrams: Among more than two hundred flares detected during these runs with the $1.25 \mathrm{~m} \mathrm{AZT-11}$ telescope of the Crimean Astrophysical Observatory and the photometer-polarimeter by Piirola (1984), several flares were registered in the total UBVRI bands. From these flares we selected those for which the pure flare radiation colour indices were determined with $\sigma<0$ m 25 at brightness maximum. In a second step, we rejected double peak flares, since their light curves may be superpositions of independent events. As a result, we obtained 7 flares with amplitudes $\Delta U$ from $3^{\mathrm{m}} 7$ to $2^{\mathrm{m}} 0$ and considered their pure flare radiation colour indices at brightness maxima, at $1-2$ minutes before and $5-8$ minutes after maxima.

To analyze their pure flare radiation we have constructed theoretical colourcolour diagrams covering the whole $U B V R I$ range. Their construction was described earlier, and the last version of such a diagram is presented in Fig. 1 (Abdul-Aziz et al. 1995). They include theoretical colours of black body radiation, emission of hydrogen plasma, both optically thin and thick in Balmer continuum, and radiation emitted by the upper layers of red dwarf stars heated by fast particles. 

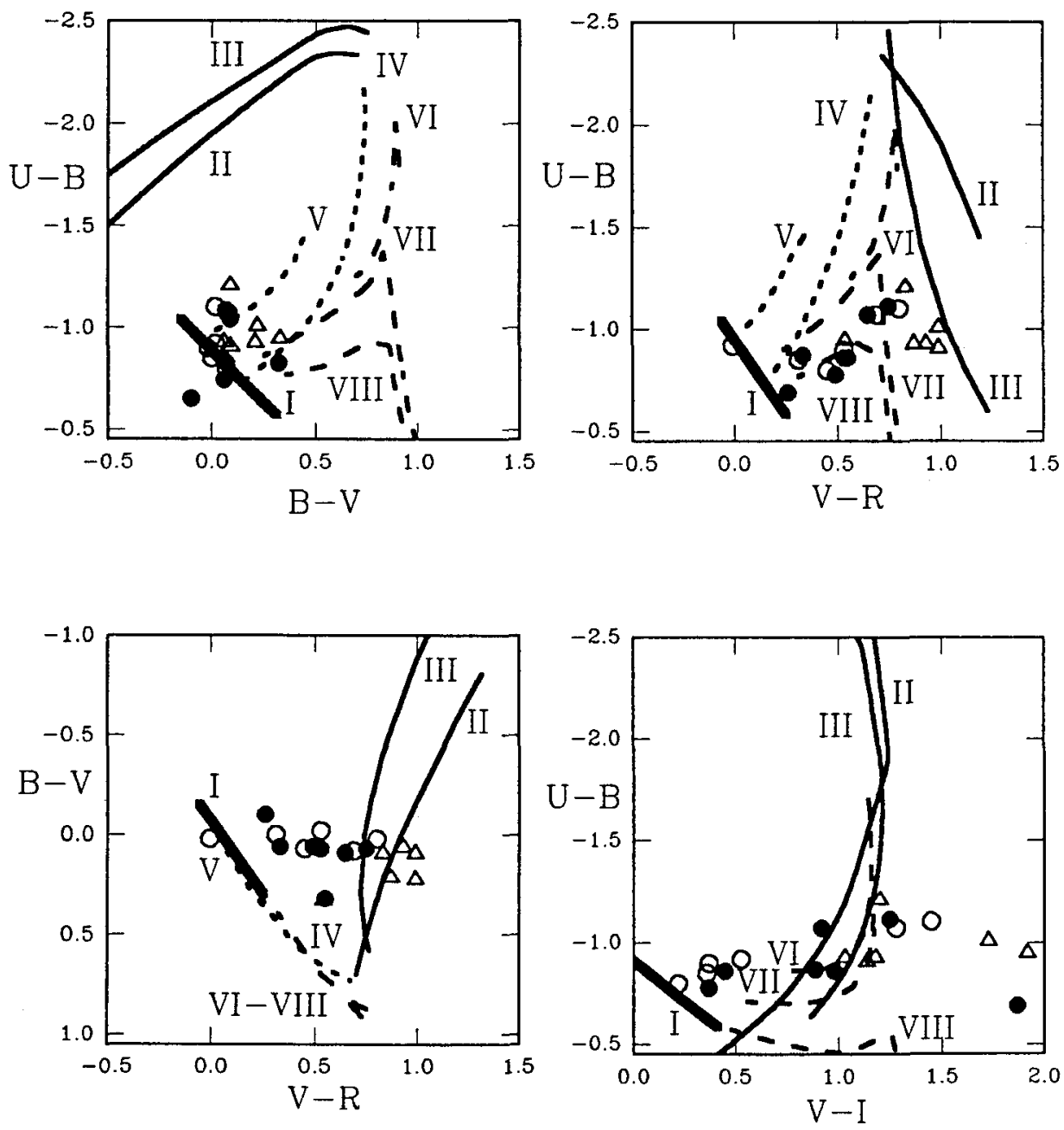

Fig. 1. Colour-colour diagrams of the pure flare radiation of powerful flares on EV Lac. Open circles - premaximum flare radiation, full circles - maximum flare radiation, and triangles - flare decay phase radiation. I - black body radiation for temperatures ranging from 6000 to $20000 \mathrm{~K}$; II and III - emission of hydrogen plasmas, optically thin in the Balmer continuum $\left(\mathrm{Ba}_{c}\right)$, with $T=10000 \mathrm{~K}$ and $n=10^{12}$ and $10^{14} \mathrm{~cm}^{-3}$, respectively; IV and V - emission of optically thick in the Ba hydrogen plasmas, optically thick in the $\mathrm{Ba}_{c}$, with $T=10000$ and $15000 \mathrm{~K}$, respectively; VI, VII and VIII - radiation emitted by upper atmospheric layers heated by fast particles of different energies. 
Fig. 1 shows that at flare maxima (full circles) the flare radiation in the $U B V$ bands is rather close to that of a blackbody. However, in the $R$ and $I$ bands we find significant deviations from such a radiation model. Examining the $(U-B, V-R$ ) diagrams, one finds a shift of the flare maximum radiation from the black body line toward the region occupied by radiation emitted by the upper layers of a stellar photosphere heated by fast particles. In the $(B-V, V-R)$ diagrams we find even larger deviations from the black body line, and the flare maximum radiation reaches the region corresponding to optically thin hydrogen plasma emission.

Multicolour measurements at premaximum phases (open circles) show that at these phases the colours of the pure flare radiation were rather close to the respective colours at subsequent flare maxima.

At the decay phase (triangles) in the $(U-B, B-V)$ and $(U-B, V-R)$ diagrams we find shifts of the pure flare radiation from the black body line towards the regions of radiation coming from hydrogen plasma and/or of heated upper atmospheric layers. However, in the $(B-V, V-R)$ diagram we see once again shifts toward the region of optically thin hydrogen plasma only.

In the $(U-B, V-I)$ diagram, the pure flare radiation is spread over the whole region from black body to hydrogen plasma or heated upper atmospheric layers, and in some cases even across the regions corresponding to any of the above considered models.

Thus, Fig. 1 permits to conclude that none of the four models considered above can alone represent the flare emission over the whole UBVRI range at any flare phase. The observations are better represented by a combination of two computed models, for instance by the superposition of short-lived black body radiation and a slowly developing emission of hydrogen plasma. However, in general, these models may be regarded only as starting blocks for a real model of flares.

2. Polarimetry: In 1989 and 1991, during the polarimetric monitoring of the star with the $2.6 \mathrm{~m}$ Shajn reflector of the Crimean Astrophysical Observatory, about ten EV Lac flares were detected. The most powerful of these was detected on September 15 at $01: 29$ UT with $\Delta U=3^{m}$. The data analysis leads to the conclusion that the polarization of the star during this powerful flare was less than $2 \%$ at $10 \mathrm{~s}$ time resolution and less than $1 \%$ at $50 \mathrm{~s}$ time resolution. Detailed discussion on all earlier polarimetric data on the UV Cet type flares permit to conclude that the published data on discoveries of flare polarization or on significantly smaller upper limits of polarization are questionable. These conclusions are described in detail by Alekseev et al. (1994).

3. Spectroscopy: During the spectroscopic monitoring of EV Lac with the CCD spectrograph of the Shajn reflector in 1992 we (1) confirmed a kinematic heterogeneity of flare matter responsible for the hydrogen emission, (2) measured a veiling of intensity jumps in the $\mathrm{TiO}$ molecular band heads by flare continuum emission, and (3) detected several noticeable emission lines near $H \beta$. These lines were registered already earlier and have been attributed to $\mathrm{HeI}$ and $\mathrm{MgI}$ emis- 
sion. However, we suggest that they are Fe II (42) emission, well known in solar flare spectra and in T Tau type star spectra. These results are in preparation (Abdul-Aziz et al. 1995).

4. Simultaneous radio monitoring: The EV Lac flare on September 10, 1993, at UT 22:32 was detected in Crimea in the $U$ band, and was monitored simultaneously by M. Kidger at the Carlos Sanchez telescope on the Canary Islands in the $K$ band. Data analysis shows that this flare with $\Delta U=3^{\mathrm{m}} 1$ did not vary noticeably in the $K$ band. Thus, the dip in the $K$ band detected during the AD Leo flare on 1984 March 28, at 03:22 UT (Rodono 1986), is not a universal feature of UV Cet type flares.

5. Simultaneous Radio monitoring: During the optical monitoring of EV Lac in 1992, a decametre radio wavelength patrol has also been carried out. As is known, decametric emission from a flare of a red dwarf star has been detected only once before: on 1963 November 3, Slee et al. (1963) detected a flare on V 371 Ori at frequencies of $19.7,410$ and $1410 \mathrm{MHz}$. However, such events were not recorded later by any other observers.

The synphase grating UTR-2 of the Radio Astronomical Institute of the Ukrainian Academy of Sciences (Braude et al. 1978), the largest decametric radio telescope in the world, was used. Radio brightness monitoring of the star was made by a beam supplied to two-channel radiometers at $25 \mathrm{MHz}$ and $20 \mathrm{MHz}$. Such a two-frequency receiver permits the discrimination of broadcasting interference and allows to estimate a bandwidth and spectral index of the detected signal. Simultaneously, a third, similar radiometer was used to monitor an area offset by $1^{\circ}$ in declination from EV Lac. Local impulse interference would be expected to occur in all beams.

Radio monitoring of EV Lac was made on September 1-6, between 21 to 26 UT each night. During these 30 hours, more than thirty bursts were detected. About half of them were identified with terrestrial interference, the rest were individual bursts or compact groups of bursts with typical durations of $1-3 \mathrm{~s}$ and $10 \mathrm{~s}$, respectively. Those bursts which could not be identified with terrestrial interferences, show a general correlation with the optical activity of the star. Indeed, if one considers the temporal intervals from 21 to 26 UT during which the radio monitoring was carried out, a ranking of nights according to the stellar optical activity from higher to lower levels is the following: September 1, 4, 3 and 2. On the other hand, the number of radio bursts detected during these nights are $9,5,2$ and 1 . During the same nights the number of radio bursts attributed to terrestrial interference are 7,5,3 and 5. In spite of small statistics, it is clear that the second distribution is noticeably more even than the first one. This general correlation permits to suggest that radio bursts coincident in time with optical flares are most probably of stellar origin. Groups of bursts were detected only during the night September $1 / 2$, when the optical activity level was high. During the other nights, with moderate and low optical activity of the star, only weak individual bursts with a duration of about $1 \mathrm{~s}$ were detected. These two types of bursts exhibit different signal intensities as well. If one supposes that 
the radio source size is equal to the stellar area with $R_{*}=0.35 R_{\odot}$, the fluxes detected correspond to brightness temperatures from $1 \cdot 10^{18}$ to $4 \cdot 10^{17} \mathrm{~K}$. These values are 2 orders of magnitudes lower than the corresponding value for the burst of V 371 Ori at $19.7 \mathrm{MHz}$ (Slee et al. 1963) and not more than 3-4 orders of magnitude higher than the strongest type III solar bursts (Melrose 1989). It is appropriate to mention here that if one considers optical flares with a mean occurrence interval of about a dozen hours both on EV Lac and on the Sun, the total optical energy of the stellar events is also 3-4 orders of magnitude higher than the respective solar flares (Gershberg 1989).

Thus, the probability seems to be high that we detected decametric radio emission from the flare star EV Lac. However, remaining doubts lead to some caution in the conclusions and require continuation of this observational program. This study is described in detail by Abranin et al. (1994).

\section{References}

Abdul-Aziz H., Abranin E.P., Alekseev I.Yu., Avgoloupis S., Bazelyan L.L., Beskin G.M., Brazhenko A.I., Chalenko N.N., Cutispoto G., Fuensalida J.J., Gershberg R.E., Kidger M.R., Leto G., Malkov Yu.F., Mavridis L.N., Pagano I., Panferova I.P., Pustil'nik L.A., Rodonò M., Seiradakis J.H., Sergeev S.G., Spencer R., Shakhovskaya N.I., Shakhovskoy D.N., 1995, (in prep.)

Abranin E.P., Alekseev I.Yu., Bazelyan L.L., Brazhenko A.I., Gershberg R.E., Shakhovskaya N.I., 1994, Kinematics and physics of celestial bodies 10, N 4, 70

Alekseev I.Yu., Gershberg R.E., Ilyin I.V., Shakhovskaya N.I., Shakhovskoy N.M., Avgoloupis S., Mavridis L.N., Seiradakis J.H., Kidger M.R., Panferova I.P., Pustil'nik L.A., 1994, A\&A 288, 502

Berdyugin A.V., Gershberg R.E., Ilyin I.V., Malanushenko V.P., Shakhovskaya N.I., Shakhovskoy N.M., Pagano I., Panferova I.P., Pustil'nik L.A., 1995, Izvestiya Krymsk. Astrophys. Obs. 89, (in press)

Braude S.Ya., Megn A.V., Ryabov B.P., Sharykin N.K., Zhouck I.N., 1978, ApSS 54, 3

Gershberg R.E., 1989, Mem. S.A. It. 60, 263

Gershberg R.E., Grinin V.P., Dyin I.V., Nesterov N.S., Shakhovskaya N.I., Getov R.G., Ivanova M.S., Panov K.P., Tsvetkov M.K., Tsvetkova A.G., Leto G., 1991a, Astron. Zh. 68,548

Gershberg R.E., Dyin I.V., Shakhovskaya N.I., 1991b, Astron. Zh. 68, 959

Gershberg R.E., Myin I.V., Rostopchina A.N., Shakhovskaya N.I., Garbuzov G.A., Pettersen B.R., Korhonen T., Avgoloupis S., Mavridis L.N., Seiradakis J.H., Konstantinova-Antova R.K., Antov A.P., Melkonyan A.S., Panferova I.P., Pustil'nik L.A., Herouni P.M., Oskanyan A.V., 1993, Astron. Zh. 70, 984

Melrose D.B., 1989, Solar Phys. 120, 369

Rodonò M., 1986, in Flare stars and related objects, L.V.Mirzoyan (ed.), Publ. House Armenian Acad. Sci., Yerevan, p. 19

Slee O.B., Solomon L.H., Patston G.E., 1963, Nature 199, 991 